\title{
Modern Approaches to Detect and Classify Comment Toxicity Using Neural Networks
}

DOI: $10.18255 / 1818-1015-2020-1-48-61$

${ }^{1}$ P. G. Demidov Yaroslavl State University, 14 Sovetskaya, Yaroslavl 150003, Russia.

MSC2020: 68T50

Received January 17, 2020

Research article

Full text in Russian

After revision February 25, 2020

Accepted February 28, 2020

The growth of popularity of online platforms which allow users to communicate with each other, share opinions about various events, and leave comments boosted the development of natural language processing algorithms. Tens of millions of messages per day are published by users of a particular social network need to be analyzed in real time for moderation in order to prevent the spread of various illegal or offensive information, threats and other types of toxic comments. Of course, such a large amount of information can be processed quite quickly only automatically. That is why there is a need to find a way to teach computers to "understand" a text written by humans. It is a non-trivial task even if the word "understand" here means only "to classify". The rapid evolution of machine learning technologies has led to ubiquitous implementation of new algorithms. A lot of tasks, which for many years were considered almost impossible to solve, are now quite successfully solved using deep learning technologies. This article considers algorithms built using deep learning technologies and neural networks which can successfully solve the problem of detection and classification of toxic comments. In addition, the article presents the results of the developed algorithms, as well as the results of the ensemble of all considered algorithms on a large training set collected and tagged by Google and Jigsaw.

Keywords: toxicity; Natural Language Processing; NLP; deep learning; word embedding; GloVe; FastText; recurrent neural networks; convolutional neural networks; CNN; LSTM; GRU

\section{INFORMATION ABOUT THE AUTHORS}

$$
\begin{array}{l|l}
\text { Sergey V. Morzhov } & \begin{array}{l}
\text { orcid.org/0000-0001-6652-3574. E-mail: smorzhov@gmail.com } \\
\text { postgraduate student. }
\end{array}
\end{array}
$$

For citation: S. V. Morzhov, "Modern Approaches to Detect and Classify Comment Toxicity Using Neural Networks", Modeling and analysis of information systems, vol. 27, no. 1, pp. 48-61, 2020. 


\section{Современные методы детектирования и классификации токсичных комментариев с использованием нейронных сетей}

С. В. Моржов ${ }^{1}$

DOI: $10.18255 / 1818-1015-2020-1-48-61$

\section{УДК 004.8}

Научная статья

Полный текст на русском языке
Получена 17 января 2020 г.

После доработки 25 февраля 2020 г. Принята к публикации 28 февраля 2020 г.

Рост популярности онлайн-платформ, позволяющих пользователям общаться друг с другом, делиться мнениями о различных событиях, оставлять комментарии, подтолкнул к развитию алгоритмов обработки естественного языка. Десятки миллионов сообщений в день, которые публикуют пользователи отдельно взятой социальной сети, необходимо анализировать в режиме реального времени или близко к тому с целью модерации, чтобы не допустить распространение различной противозаконной или оскорбительной информации, угроз и других видов токсичных комментариев. Разумеется такой большой объем информации может быть обработан достаточно быстро только автоматически. Возникает необходимость научить компьютер «понимать» текст, написанный человеком, что является нетривиальной задачей, пусть даже под «пониманием» текста подразумевается лишь его классификация. Бурное развитие технологий машинного обучения обусловило повсеместное внедрение новых алгоритмов. Многие задачи, в том числе и задачи обработки естественного языка, которые долгие годы считалось практически невозможно решить, сейчас вполне успешно решаются с использованием технологий глубокого обучения. В данной статье будут рассмотрены алгоритмы, построенные с использованием технологий глубокого обучения и нейронных сетей, позволяющие успешно решать задачу распознавания и классификации токсичных комментариев. Помимо этого, в статье будут приведены результаты тестирования как разработанных алгоритмов, так и ансамбля данных алгоритмов на большой обучающей выборке, собранной и размеченной специалистами компаний Google и Jigsaw.

Ключевые слова: токчисность; обработка естественного языка; NLP; глубокое обучение; векторное представление слов; GloVe; FastText; реккурентные нейронные сети; сверточные нейронные сети; CNN; LSTM; GRU

\section{ИНФОРМАЦИЯ ОБ АВТОРАХ}

Сергей Владимирович Моржов | orcid.org/0000-0001-6652-3574. E-mail: smorzhov@gmail.com аспирант.

Для цитирования: S. V. Morzhov, "Modern Approaches to Detect and Classify Comment Toxicity Using Neural Networks", Modeling and analysis of information systems, vol. 27, no. 1, pp. 48-61, 2020.

(C) Моржов С. B., 2020

Эта статья открытого доступа под лицензией CC BY-NC-ND license (http://creativecommons.org/licenses/by-nc-nd/4.0/). 


\section{Введение}

Задача обработки естественного языка уже многие годы является привлекательной целью исследований, так как решение ее в общем виде позволит создать естественно-языковой интерфейс, что существенно упростит и расширит сферы взаимодействия человека с компьютером. Само по себе понимание естественного языка - нетривиальная задача, считающаяся AI-полной, потому как распознавание естественного языка требует больших знаний об окружающем мире и возможности о взаимодействия с ним. Однако, при решении отдельных классов задач, например, классификации или анализа тональности текста, в последнее время был достигнут большой прогресс благодаря развитию нейросетевых алгоритмов, а также появлению высокопроизводительных процессоров и графических карт. Это позволило использовать глубокие нейронные сети для решения различных задач, связанных с обработкой естественного языка, которые ранее не могли быть успешно решены с применением классических алгоритмов.

В последнее время широкое распространение получили онлайн-платформы, позволяющие пользователям различные виды взаимодействия друг с другом, в том числе посредством обмена сообщениями. Различные социальные сети, платформы онлайн-игр, приложения для обмена фотографиями и видео, новостные порталы встраивают в свои продукты чаты, реализуют возможность оставлять комментарии, позволяют пользователям общаться друг с другом. Данный функционал уязвим перед многими видами Интернет-преступлений, среди которых можно выделить: личные оскорбления и угрозы, различные виды пропаганды, мошенничество, реклама незаконных товаров и услуг. Противоправные и токсичные комментарии должны удаляться, хотя, бесспорно, лучшим вариантом будет служить возможность запрета их публикации. Это предусматривает наличие достаточно быстрых и эффективных алгоритмов, способных в режиме реального времени обрабатывать все сообщения пользователей.

Компанией Jigsaw, занимающейся проблемами безопасности в Интернете, совместно с Google проводился конкурс [1], целью которого было создание алгоритма, решающего задачу детектирования токсичных комментариев. Это говорит об актуальности, а также низком уровне исследований данной проблемы, так как опубликованные алгоритмы, позволяющие решать поставленную задачу (см. [2-5]), имеют недостаточную по мнению организаторов конкурса аккуратность предсказаний.

В данной статье будут представлены новые алгоритмы, позволяющие решать задачу детектирования и классификации токсичных комментариев. Также будут приведены сравнительные результаты тестирования разработанных алгоритмов и некоторых существующих алгоритмов, решающих эту задачу. Помимо этого, в статье содержится ряд замечаний касательно проведения дальнейшей работы по улучшению качества предсказаний представленных алгоритмов.

\section{1. Анализ обучающей выборки}

Для решения поставленной задачи использовалась обучающая выборка, подготовленная и размеченная специалистами компаний Google и Jigsaw. Данная выборка составлена из комментариев со страниц обсуждения статей Википедии. Размер предоставленной тренировочной части выборки составляет примерно 160 тысяч комментариев. Также предоставлена тестовая выборка, содержащая примерно 154 тысячи комментариев.

Обучающая выборка размечена следующим образом. Каждому комментарию соответствует шесть меток: токсичность (toxic), сильная токсичность (severe toxic), непристойность (obscene), угроза (threat), оскорбление (insult), ненависть к личности (identity hate). Метки принимают значение 1, если в комментарии есть данный тип токсичности, 0 - иначе. Случай, когда все метки нулевые означает, что комментарий не токсичен. Может быть так, что один комментарий содержит несколько типов токсичности. Разметка данных проводилась с помощью краудсорсинга (метки выставляли разные люди), а значит возможно наличие различных ошибок. 
Тренировочная и тестовая выборки не содержат пропущенных значений. Тренировочная выборка крайне не сбалансирована - есть большой перекос в сторону «чистых», неоскорбительных, комментариев, что логично, так как в реальной жизни таких комментариев тоже обычно больше. Количество меток для каждого класса представлено на рисунке 1. Длины комментариев также сильно различаются (см. рисунок 2) и имеют следующие характеристики: минимальная длина -2 символа, максимальная - 5000 символов, математическое ожидание - 394.07, среднеквадратическое отклонение - 590.72 .

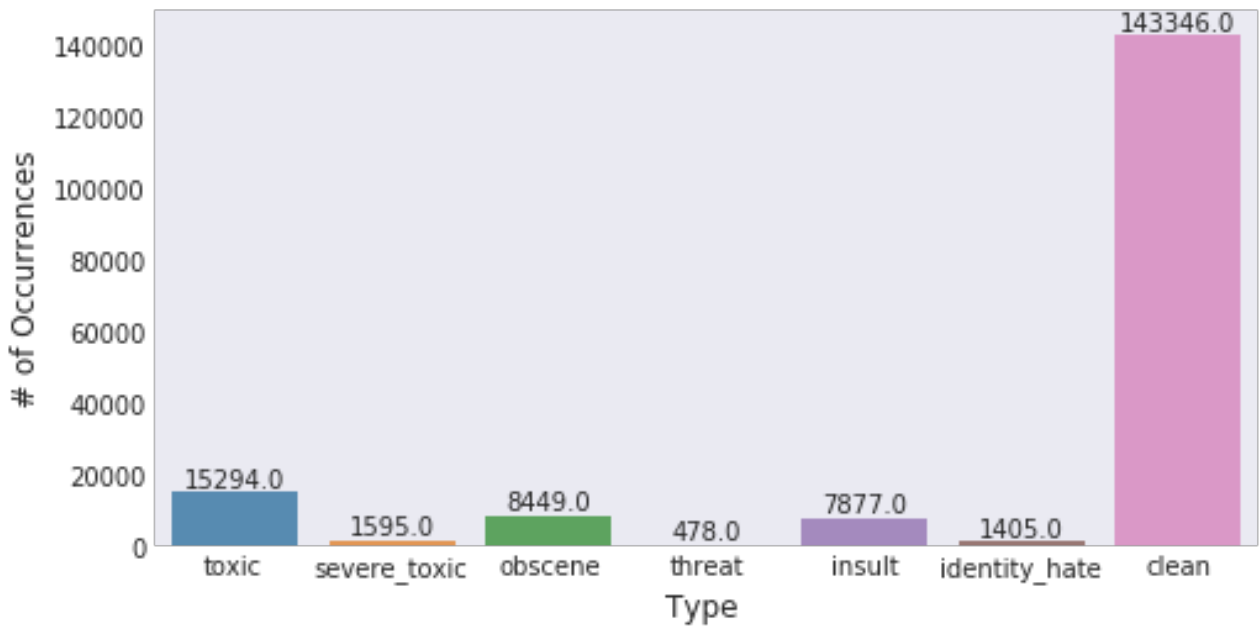

Fig. 1. Number of labels of each class

Рис. 1. Количество меток каждого класса

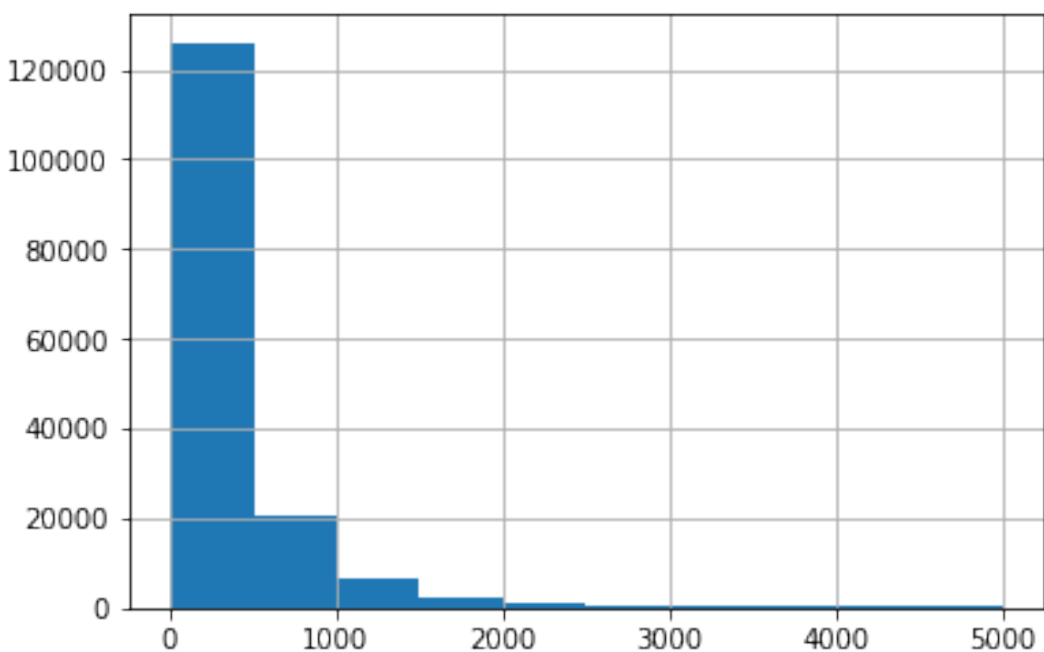

Fig. 2. Comment length distribution

Рис. 2. Распределение длины комментариев

Как уже было сказано выше, комментарий может иметь несколько меток, равных 1. Количество комментариев в зависимости от количества меток, равных 1, приведено на рисунке 3. Помимо этого, стоит также рассмотреть корреляцию между метками, равными 1 (см. рисунок 4). Исходя из анализа матрицы корреляции, можно сделать вывод, что класс «tохіс» сильно коррелирует с классами «obscene» и «insult» $(0.68$ и 0.65), класс «insult» и «obscene» также имеют высокий индекс 
корреляции (0.74). Интересным является индекс корреляции между классами «toxic» и «severe toxic», оказавшийся достаточно низким (0.31). В силу того, что количество комментариев, принадлежащих к этим классам сильно различается, стоит воспользоваться другим методом оценки корреляции.

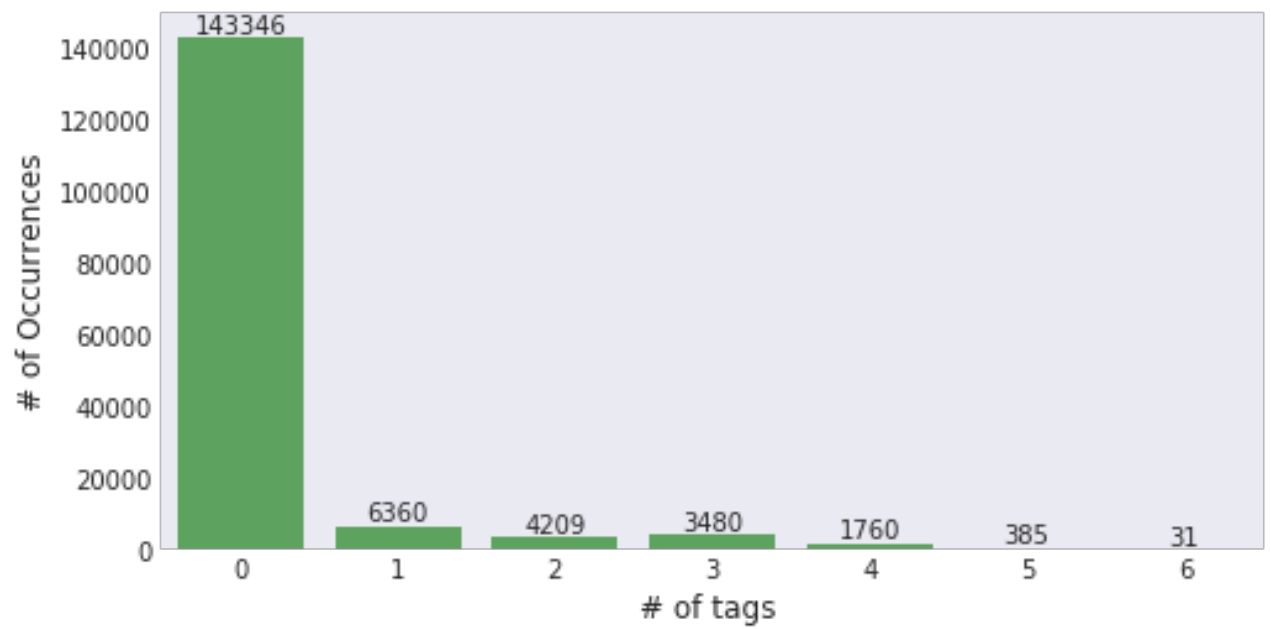

Fig. 3. Number of comments depending on the number of tags

Рис. 3. Количество комментариев в зависимости от количества меток

\begin{tabular}{|c|c|c|c|c|c|c|c|}
\hline toxic & 1 & 0.31 & 0.68 & 0.16 & 0.65 & 0.26 & -0.97 \\
\hline severe_toxic & 0.31 & 1 & 0.4 & 0.13 & 0.38 & 0.19 & -0.3 \\
\hline obscene & 0.68 & 0.4 & 1 & 0.15 & 0.74 & 0.29 & -0.7 \\
\hline threat & 0.16 & 0.13 & 0.15 & 1 & 0.16 & 0.12 & -0.17 \\
\hline insult & 0.65 & 0.38 & 0.74 & 0.16 & 1 & 0.33 & -0.68 \\
\hline identity_hate & 0.26 & 0.19 & 0.29 & 0.12 & 0.33 & 1 & -0.27 \\
\hline none & -0.97 & -0.3 & -0.7 & -0.17 & -0.68 & -0.27 & 1 \\
\hline & 这 & 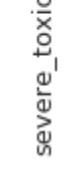 & 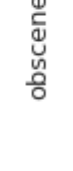 & ڤั & $\begin{array}{l}\frac{ \pm}{\vec{J}} \\
\stackrel{\text { }}{\cong}\end{array}$ & 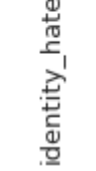 & 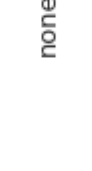 \\
\hline
\end{tabular}

Fig. 4. Toxic labels correlation matrix

Рис. 4. Матрица корреляции меток

Рассмотрим таблицу сопряженности для метки «toxic» и всех остальных меток (см. таблицу 1). Можно отметить, что комментарии, отмеченные как «severe toxic», всегда будет также отмечены как «toxic». Другие классы, похоже, также являются подмножествами класса «toxic» за некоторыми исключениями. 
Table 1. Cross tabulation for the "toxic" tag

Таблица 1. Таблица сопряженности для метки «toxic»

\begin{tabular}{|c|c|c|c|c|c|c|c|c|c|c|}
\hline & \multicolumn{2}{|c|}{ severe toxic } & \multicolumn{2}{c|}{ obscene } & \multicolumn{2}{c|}{ threat } & \multicolumn{2}{c|}{ insult } & \multicolumn{2}{c|}{ identity hate } \\
\hline $\begin{array}{c}\text { severe } \\
\text { toxic }\end{array}$ & 0 & 1 & 0 & 1 & 0 & 1 & 0 & 1 & 0 & 1 \\
\hline toxic & & & & & & & & & & \\
\hline 0 & 144277 & 0 & 143754 & 523 & 144248 & 29 & 143744 & 533 & 144174 & 103 \\
\hline 1 & 13699 & 1595 & 7368 & 7926 & 14845 & 449 & 7950 & 7344 & 13992 & 1302 \\
\hline
\end{tabular}

Существует еще одна важная особенность, на которую необходимо обратить внимание: количество уникальных слов в каждом комментарии. Данная характеристика может помочь при решении поставленной задачи, так как нетрудно заметить, что авторы токсичных комментариев не очень изобретательны в своей лексике. Иными словами, необходимо проверить следующую гипотезу: есть ли корреляция между различными характеристиками комментариев, связанными с количеством уникальных слов и токсичностью комментария. Нетрудно отметить, что существуют заметные сдвиги в среднем числе слов и количестве уникальных слов в чистых и токсичных комментариях (см. рисунок 5). Кроме этого, если рассмотреть график на рисунке 6, то можно заметить, что рядом с отметкой 0 - 10\% имеется выпуклость, указывающая на большое количество токсичных комментариев, которые содержат небольшое количество разнообразных слов.

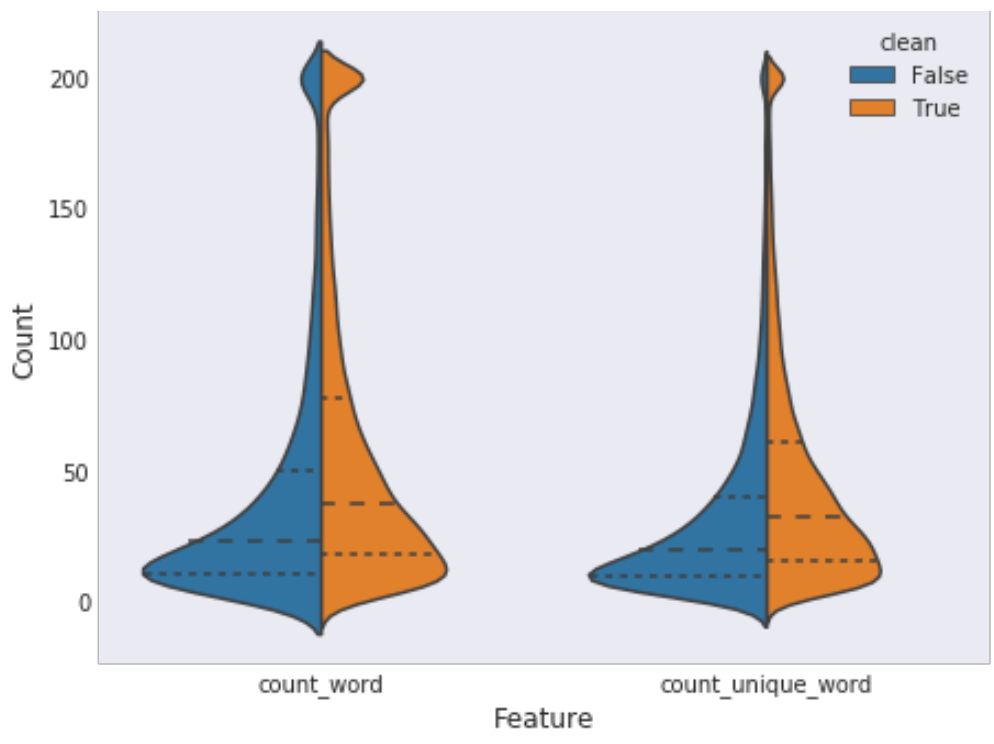

Fig. 5. Absolute number of words and number of unique words

Рис. 5. Абсолютное количество слов и количество уникальных слов

Все эти наблюдения необходимо учитывать при построении нейросетевых алгоритмов для решения задачи детектирования и классификации токсичных комментариев. Данные статистические особенности обучающей выборки помогут в дальнейшем при интерпретации результатов, а также их можно использовать для отладки и улучшения аккуратности предсказаний разработанных моделей. 


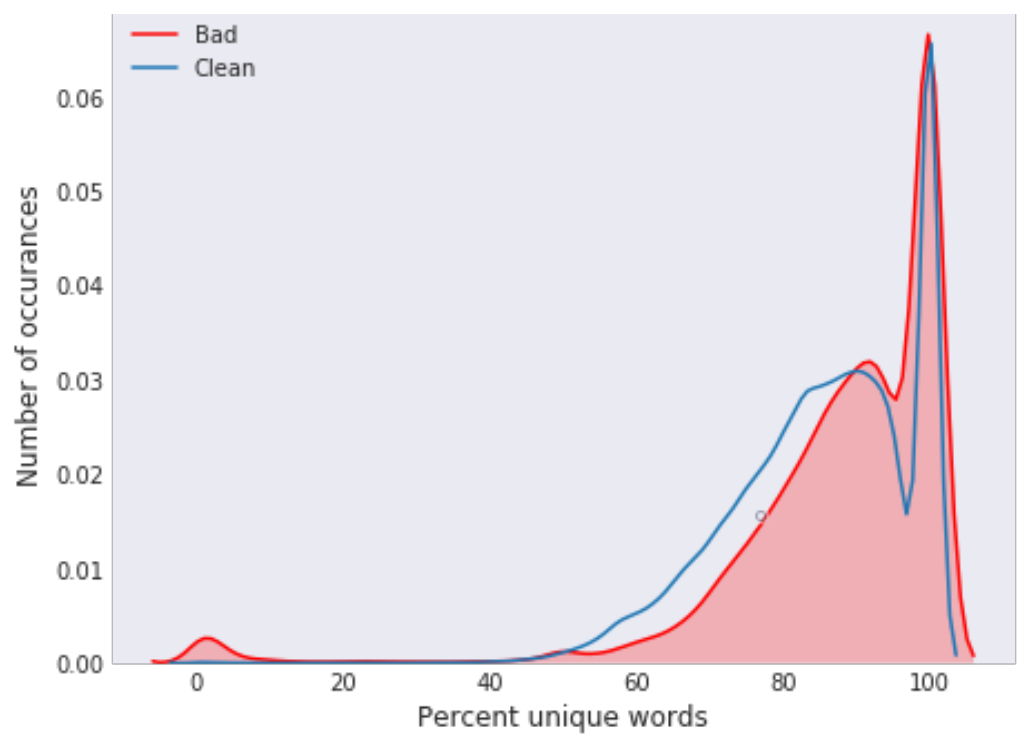

Fig. 6. Percentage of unique words in the total number of words in a comment

Рис. 6. Процент уникальных слов от общего количества слов в комментарии

Помимо статистических особенностей обучающей выборки необходимо также рассмотреть особенности текстовых данных. Комментарии преимущественно написаны на английском языке (в выборке присутствуют комментарии на других языках, но их количество составляет менее $0.1 \%$ от общего числа). Многие комментарии содержат эмодзи, избыточное количество пунктуационных знаков, веб-ссылки, числа, особые начертания слов, грамматические ошибки. Некоторые грамматические ошибки были допущены авторами специально, чтобы «замаскировать» оскорбительные или нецензурные высказывания. Все это излишне расширяет размер словаря, что в свою очередь усложняет анализ комментариев, поэтому подготовка данных из обучающей и тренировочной выборки является важным этапом при решении поставленной задачи.

\section{2. Подготовка данных}

Для решения задачи использовалась двухэтапная подготовка данных. На первом этапе осуществлялись базовые, наиболее простые манипуляции с ними:

1. Приведение текста к нижнему регистру.

2. Удаление кусков html-кода, которые присутствуют в некоторых комментариях.

3. Преобразование подстроки вида «w h a t a n i c e d a y» к «what a nice day». Данный вид искажения текста часто используется для маскировки нецензурных слов.

4. Удаление ссылок, ір-адресов.

5. Удаление чисел и цифр.

6. Удаление всей пунктуации, кроме «'», «.», «!», «?». При этом также удалялись дублирующиеся знаки, то есть подстрока вида «!!!!!» приводилась к «!».

7. Замена знаков окончания предложения на специальные токены. «!» заменялся на « exclmrk », «?» - на « qstmrk », «.»- на « eosmkr ». Это было сделано для того, чтобы не потерять информацию об этих знаках на этапе трансформации текста в векторное представление. 
На втором этапе проводились более трудоемкие операции исправления и очистки текста.

1. Замена эмодзи на соответствующие слова («:-(» на «sad» и т.д.).

2. Расшифровка некоторых сокращений («won't» на «will not», «'ll» на « will» и т.д.).

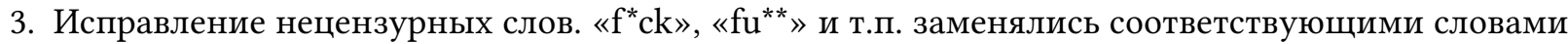
без звездочек.

4. Исправление иных грамматических ошибок ${ }^{1}$.

Обработку данных было решено разбить на две части также для того, чтобы уменьшить взаимную корреляцию моделей на этапе ансамбля. В процессе обучения каждая модель обучалась на данных, прошедших либо только первый этап обработки, либо оба этапа, что повышало вариативность предсказаний.

\section{3. Модели}

Для решения задач анализа текста хорошо себя зарекомендовали рекуррентные нейронные сети (РНС). Рекуррентные нейронные сети (РНC) используют идею обработки последовательной информации. Термин «рекуррентные» используется для того, чтобы показать, что нейронные сети выполняют одну и ту же задачу для каждого экземпляра последовательности, так что выходные данные зависят от предыдущих вычислений и результатов. Как правило, вектор фиксированного размера создается для представления последовательности путем подачи лексем одной за другой в рекуррентный блок. В некотором смысле, РНС «запоминает» предыдущие вычисления и используют эту информацию в текущей обработке. С задачами классификации текста лучше всего справляются такие разновидности PHC, как LSTM [6] и GRU [7], поэтому при построении моделей для решения поставленной задачи детектирования и классификации токсичных комментариев было решено использовать именно их.

\section{1. Векторное представление слов}

При решении любой задачи NLP неизбежно встает проблема представления слов в понятном для компьютера виде. Наиболее тривиально данную задачу можно решить следующим образом: закодируем все слова словаря цифрами по порядку. Так как натуральный ряд бесконечен, то не составит труда перенумеровать все слова. Однако, у этой идеи есть существенный недостаток. Согласно гипотезе лингвиста Фердинанда де Соссюра, буквенное написание слова совершенно не связано с его смыслом. Возьмем, к примеру, слова «петух», «курица», «цыпленок». В словаре они находятся далеко друг от друга, хотя очевидно обозначают самца, самку и детеныша одного вида птиц. Таким образом, можно выделить два вида близости слов: лексическую и семантическую. Пример с курицей показывает, что они не всегда совпадают. Для наглядности можно привести обратный пример лексически близких, но семантически далеких слов: «зола» и «золото».

Существует гипотеза, согласно которой слова, имеющие близкое значение, как правило употребляются в текстах в близких по смыслу контекстах. Отсюда вытекает, что семантическая близость слов является важным признаком, который необходимо сохранить при представлении слов в понятном для компьютера виде.

Одним из способов решения поставленной задачи является сопоставление словам (и, возможно, фразам) из некоторого конечного фиксированного словаря размера $N$ векторов из $\mathbb{R}^{n}, n<<$. Данная техника называется векторное представление слов (англ. word embedding). Так как векторы должны отражать семантическую близость слов, то пусть близкие векторы (например, по косинусной мере), обозначают близкие по смыслу слова.

\footnotetext{
${ }^{1}$ Данный вид преобразования в конечном итоге решено было не использовать из-за слишком длительной обработки в силу размера корпуса.
} 
Векторное представление слов часто используется в качестве первого слоя, преобразующего текст на естественном языке в вид, пригодный для дальнейшей обработки какой-либо моделью глубокого обучения. Для данной задачи были выбраны две модели генерации векторного представления слов: GloVe [8] и FastText [9].

\subsection{Bi-GRU-LSTM}

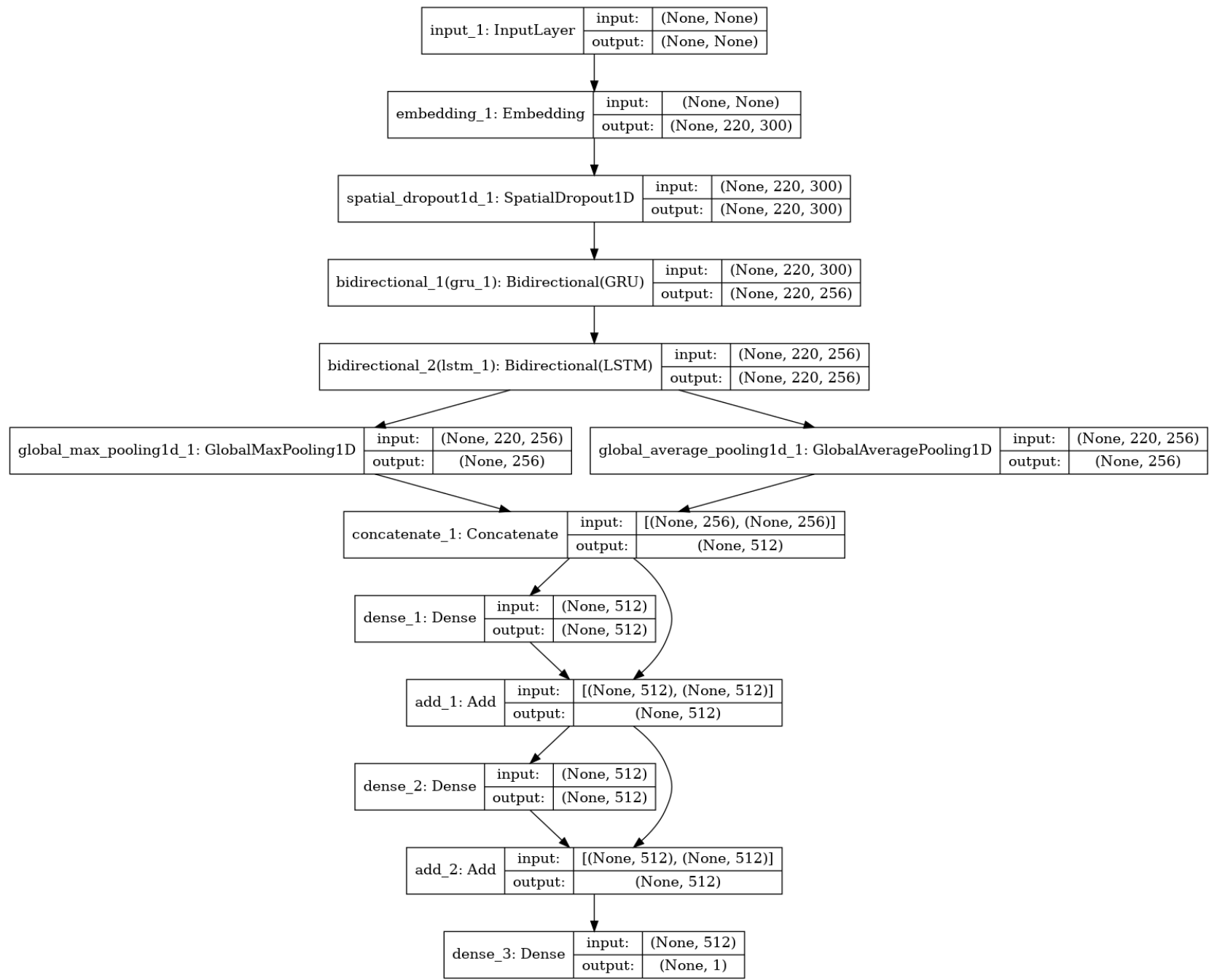

Fig. 7. The architecture of Bi-GRU-LSTM model

Рис. 7. Архитектура модели Bi-GRU-LSTM

Модель, сочетающая в себе стекинг GRU и LSTM слоев, была выбрана в качестве базовой для решения задачи классификации токсичных комментариев [10]. Она достаточно глубока, чтобы выделить необходимое количество признаков из не очень большой тренировочной выборки (всего порядка 140000 комментариев, принимая во внимание использование кросс-валидации на 10 частях). Архитектура модели Bi-GRU-LSTM представлена на рисунке 7.

\subsection{Bi-GRU с механизмом внимания}

Данная модель похожа на Bi-GRU-LSTM, за тем исключением, что здесь последовательно используются два слоя GRU и механизм внимания [11]. В оригинальной статье механизм внимания использовался на примере задачи машинного перевода. Однако ничего не мешает применить его 
и к задаче классификации [12]. По своей сути механизм внимания - это не что иное, как усовершенствованный механизм кодеров-декодеров. Использование GRU или LSTM позволяет сохранить информацию о структуре последовательности, при этом не позволяя назначать различные веса элементам полученных последовательностей. Очевидно, что в задаче классификации токсичных комментариев различные слова будут иметь разную значимость: нецензурные слова должны иметь больший вес, являясь хорошим сигналом, что комментарий вероятно токсичный. Ни LSTM, Hи GRU не способны моделировать подобное в отличие от механизма внимания. Иными словами, механизм внимания позволяет присвоить различные веса каждому элементу обрабатываемой последовательности. Чем вес больше, тем важнее данное слово, следовательно, данному слову нужно уделить больше внимания.

Архитектура модели Bi-GRU-LSTM представлена в Приложении А на странице 59.

\section{4. Асимметричный CNN-LSTM}

Данная модель является усложнением модели Bi-GRU с механизмом внимания. Были добавлены несколько сверточных слоев [13] для выделения признаков перед первым рекуррентным блоком.

Архитектура данной модели представлена в Приложении В на странице 60.

\section{4. Результаты тестирования}

В качестве метрики оценки аккуратности предсказаний разработанных моделей было решено использовать усредненный по типам токсичности ROC AUC, то есть среднее арифметическое ROC AUC (Avg. ROC AUC) для каждого класса в отдельности.

В таблице 2 приведены результаты работы различных моделей на подготовленных данных, полученных согласно описанному ранее алгоритму очистки (использовалась двухэтапная подготовка). Во второй колонке содержится среднее арифметическое Avg. ROC AUC по всем 10 фолдам.

Table 2. Comparison of the results of different toxic comments classification models
Таблица 2. Сравнение результатов работы различных моделей для классификации токсичности комментариев

\begin{tabular}{|c|c|c|}
\hline Модель & $\begin{array}{c}\text { 10-fold cross-validation Avg. } \\
\text { ROC AUC }\end{array}$ & Test Avg. ROC AUC \\
\hline CNN [2] & 0.9236 & 0.9120 \\
LSTM [3] & 0.9545 & 0.9492 \\
LSTM-CNN [4] & 0.9790 & 0.9778 \\
\hline Bi-GRU-LSTM & 0.9887 & 0.9849 \\
Bi-GRU с механизмом внимания & 0.9888 & 0.9848 \\
Асимметричный CNN-LSTM & 0.9910 & 0.9836 \\
\hline Ансамбль & 0.9889 & 0.9870 \\
\hline
\end{tabular}

В ансамбль были включены все модели, представленные в данной статье, с векторным представлением слов GloVe и FastText. Таким образом, в ансамбль были включены 6 моделей. Помимо этого использовалась техника, называемая «seed averaging», заключающаяся в запуске одной модели с инициализацией генератора псевдослучайных чисел разными значениями. Полученные таким образом предсказания усреднялись. 


\section{5. Заключение}

Технологии глубокого обучения позволяют минимизировать участие человека в разработке алгоритмов, поскольку создание признаков, свойственных конкретной задаче, автоматизируется. В данной статье было показано, как с их помощью можно решить задачу детектирования и классификации токсичных комментариев. Полученные результаты как ансамбля, так и каждой разработанной модели в отдельности превзошли результаты из ранее опубликованных работ по данной тематике, что говорит об успешности проведенных исследований.

Дальнейшее совершенствование аккуратности предсказания моделей может быть достигнуто при использовании техники аугментации, т.е. увеличения размера тренировочной выборки. Это можно сделать, например, используя технику машинного перевода. Перед каждой эпохой во время тренировки модели можно выбирать некоторое подмножество комментариев из обучающей выборки, перевести их на какой-нибудь язык, например, немецкий, затем перевести полученные комментарии обратно на английский. Такое преобразование не должно сильно исказить смысл комментариев, но при этом увеличит размер выборки. Существует также более простой вариант увеличения тренировочной выборки. Он состоит в следующем: на этапе обучения перед каждой эпохой можно выбрать некоторое подмножество комментариев из обучающей выборки, часть из которых будет склеена друг с другом. Таким образом, будут получены новые, более длинные комментарии. Метки для них можно назначить путем объединения множества меток исходных комментариев.

Также можно попробовать совместить технологии глубокого обучения с деревьями решений и градиентным бустингом [14]. В ходе подготовки и очистки данных некоторая часть информации была потеряна. Ее можно восстановить вручную, создав новые признаки, вектор из которых, совместно с вектором предсказаний разработанных моделей, следует использовать для поиска более оптимальных предсказаний. К таким новым признакам можно отнести следующие: количество знаков восклицания (токсичные комментарии обычно содержат много знаков восклицания), количество вопросительных знаков, количество грамматических ошибок, длина комментария, количество нецензурных слов и т.п. Подобное усложнение модели не должно серьезно сказаться на времени обучения и трудоемкости при использовании, но согласно проведенным грубым оценкам, способно повысить качество предсказаний примерно на $0.001-0.002$ по выбранной метрике Avg. ROC AUC. 


\section{Приложение A Bi-GRU с механизмом внимания}

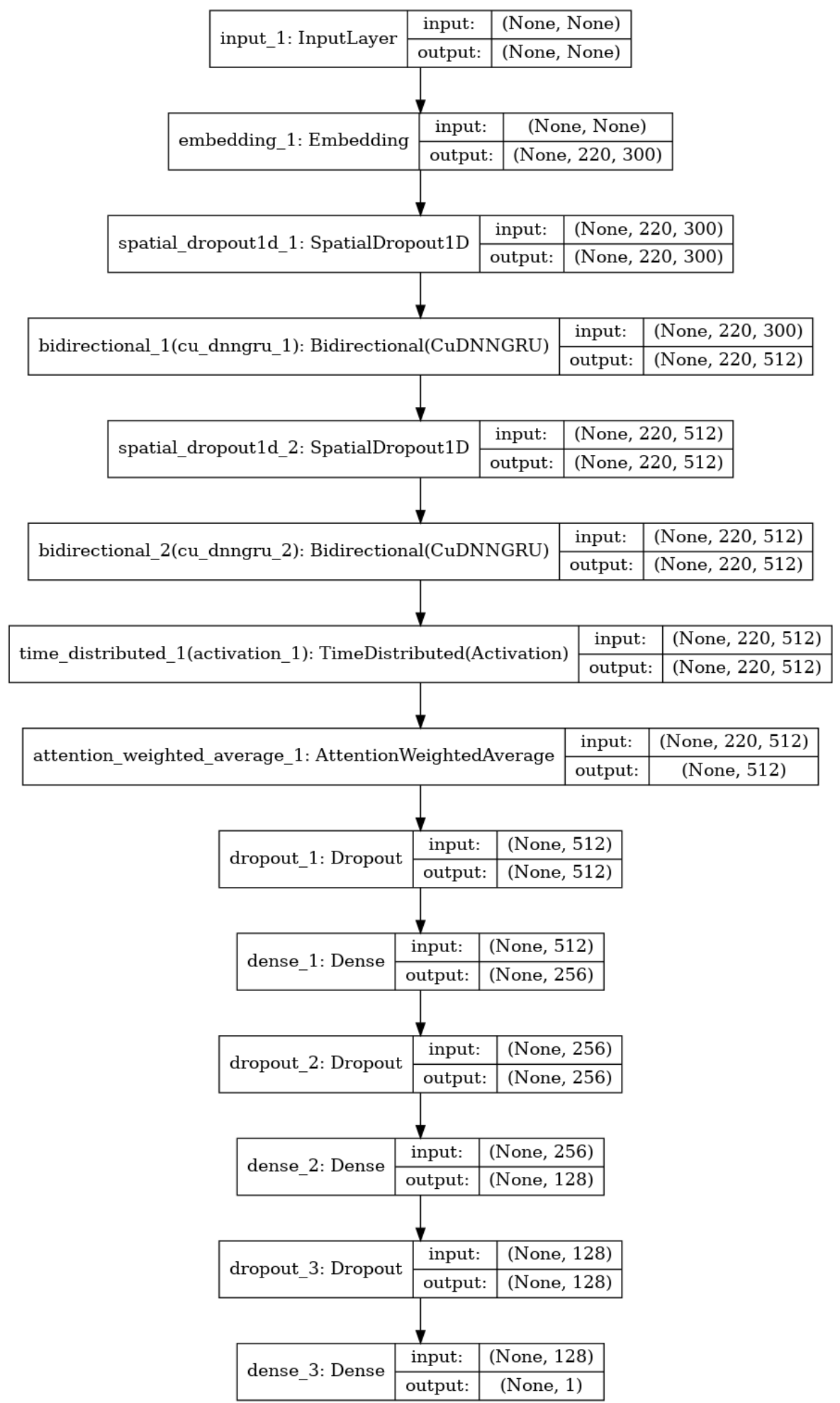

Fig. 8. The architecture of Bi-GRU with attention mechanism model
Рис. 8. Архитектура модели Bi-GRU с механизмом внимания 


\section{Приложение В Асимметричный CNN-LSTM}

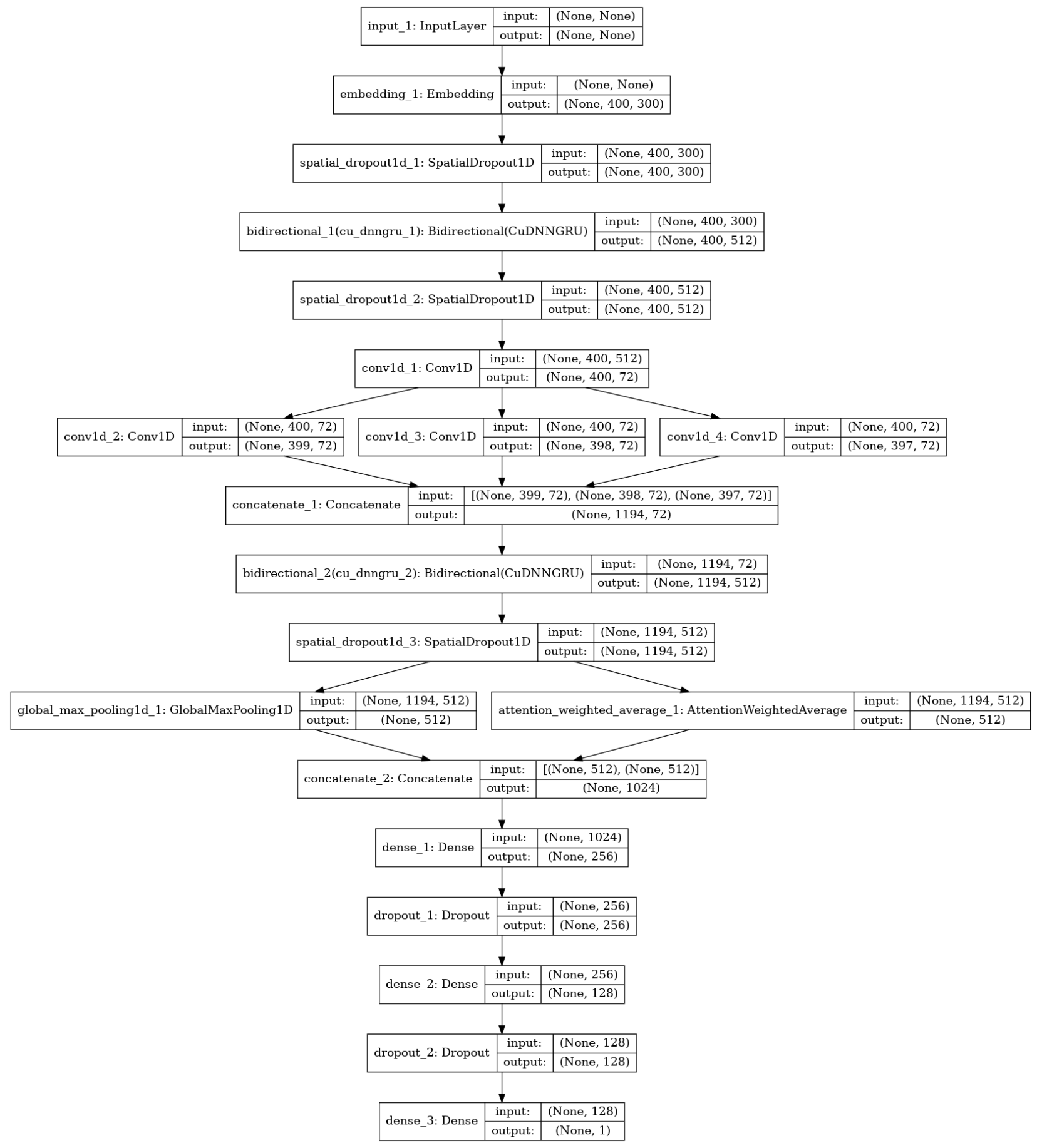

Fig. 9. The architecture of asymmetric CNN-LSTM model
Рис. 9. Архитектура асимметричной CNN-LSTM модели 


\section{References}

[1] Toxic Comment Classification Challenge. [Online]. Available: https://www.kaggle.com/c/jigsaw-toxiccomment-classification-challenge/overview.

[2] S. V. Georgakopoulos, S. K. Tasoulis, A. G. Vrahatis, and V. P. Plagianakos, "Convolutional neural networks for toxic comment classification", in Proceedings of the 10th Hellenic Conference on Artificial Intelligence, 2018, pp. 1-6. arXiv: https://arxiv.org/pdf/1802.09957.pdf.

[3] M. Kohli, E. Kuehler, and J. Palowitch, Paying attention to toxic comments online. [Online]. Available: https://web.stanford.edu/class/archive/cs/cs224n/cs224n.1184/reports/6856482.pdf.

[4] T. Chu, J. K., and M. Wang, Comment Abuse Classification with Deep Learning. [Online]. Available: https://web.stanford.edu/class/archive/cs/cs224n/cs224n.1174/reports/2762092.pdf.

[5] K. Khieu and N. N., Detecting and Classifying Toxic Comments. [Online]. Available: https://web. stanford.edu/class/archive/cs/cs224n/cs224n.1184/reports/6837517.pdf.

[6] S. Hochreiter and J. Schmidhuber, "Long short-term memory", Neural computation, vol. 9, no. 8, pp. 1735-1780, 1997.

[7] K. Cho, B. Van Merriënboer, C. Gulcehre, D. Bahdanau, F. Bougares, H. Schwenk, and Y. Bengio, "Learning phrase representations using RNN encoder-decoder for statistical machine translation", arXiv preprint arXiv:1406.1078, 2014.

[8] J. Pennington, R. Socher, and C. Manning, "Glove: Global vectors for word representation", in Proceedings of the 2014 conference on empirical methods in natural language processing (EMNLP), 2014, pp. 1532-1543.

[9] A. Joulin, E. Grave, P. Bojanowski, and T. Mikolov, "Bag of tricks for efficient text classification", Proceedings of the 15th Conference of the European Chapter of the Association for Computational Linguistics, vol. 2, pp. 427-431, 2017.

[10] J. Chung, C. Gulcehre, K. Cho, and Y. Bengio, "Empirical evaluation of gated recurrent neural networks on sequence modeling”, arXiv preprint arXiv:1412.3555, 2014.

[11] D. Bahdanau, K. Cho, and Y. Bengio, "Neural machine translation by jointly learning to align and translate", arXiv preprint arXiv:1409.0473, 2014.

[12] Z. Yang, D. Yang, C. Dyer, X. He, A. Smola, and E. Hovy, "Hierarchical attention networks for document classification”, in Proceedings of NAACL-HLT, 2016, pp. 1480-1489. [Online]. Available: https://www. cs.cmu.edu/\%5C\%20./hovy/papers/16HLT-hierarchical-attention-networks.pdf.

[13] M. Hughes, I. Li, S. Kotoulas, and T. Suzumura, "Medical text classification using convolutional neural networks”, Stud Health Technol Inform, vol. 235, pp. 246-50, 2017.

[14] K. Kowsari, K. Jafari Meimandi, M. Heidarysafa, S. Mendu, L. Barnes, and D. Brown, “Text classification algorithms: A survey", Information, vol. 10, no. 4, p. 150, 2019. 